\title{
Minimizing Power Losses in Distribution Systems Using GA in Planning and Operation: A Case Study for DGs and SCs
}

\author{
Doan Duc Tung ${ }^{1 *}$, Fabio Mottola ${ }^{2}$ and Alfredo Testa ${ }^{3}$ \\ ${ }^{1}$ Department of Engineering and Technology, Quy Nhon University, \\ Binh Dinh 820000, Vietnam \\ ${ }^{2}$ Department of Electrical Engineering and Information Technology of the \\ University of Naples Federico II, Naples 80125, Italy \\ ${ }^{3}$ Department of Industrial and Information Engineering, University of Campania, \\ L. Vanvitelli, Aversa (CE) 80017, Italy \\ 1doanductung@qnu.edu.vn,2fmottola@unina.it, ${ }^{3}$ alfredo.testa@unicampania.it
}

\begin{abstract}
This paper proposes a relevant method to plane the optimal location and size of the multiple distributed generators (DGs) and shunt capacitors (SCs) as well as the optimal operating power factor for each DG in the distribution systems. The proposed method is aimed at minimizing the power losses. Based on Genetic Algorithm (GA), the proposed method is developed to solve the optimization problem applying the nonlinear objective function and considering the parameter values as the placement and size of DGs and SCs and the power factor of DGs for minimizing power losses of the system subjected to both equality and inequality constraints. In order to check the feasibility, the outlined method is analyzed and tested on IEEE 37-node radial distribution system and the results compared with other tested cases based on the total power losses and the voltage profile at nodes.
\end{abstract}

Keywords: Genetic Algorithm (GA), distributed generator (DG), shunts capacitor $(S C)$, power loss minimization, voltage profile

\section{Introduction}

Nowadays, the integration of distributed generators (DGs) in the distribution network is the most economical solution to meet the increased electricity demand due to load growth. If DGs are properly placed at optimal location and optimally sized, it can be proved its usefulness in providing the reduction in power losses and improving the voltage profile and power quality levels of the network. On the other hand, the distribution network will be impacted negatively when DGs are connected. If the DGs are misused or misplaced, they may easily cause degradation of power quality and reliability levels as well as losses may increase; as a result, the voltage regulation is unlikely to be effective [1-2].

The DG's placement and sizing are mostly determined using the optimization algorithms, which are widely investigated in the literature from different perspectives. In Refs. [3-4], the Kalman filter algorithm has been used to minimize power losses. The authors in Ref. [5] have proposed a probabilistic power flow combined with embedded genetic algorithm to solve the problem of the total costs minimization. In this Ref., the costs associating with the installation of DGs in a distribution system are the investment cost, operating cost, maintenance cost, capacity adequacy cost, and network loss cost. The method based on a modified teaching-learning optimization algorithm is introduced in

*Corresponding Author 
Ref. [6] to minimize total power losses. The use of a local particle swarm optimization (PSO) was discussed in [7].

Many optimization algorithms for multi-objective approaches were also proposed in recent literature. The Multi-objective optimization including of the voltage stability, power losses, and network voltage variations has been proposed in Ref. [8]. In [9], the authors proposed an advanced Pareto-front non-dominated sorting multi-objective particle swarm optimization method and the optimization problem has considered two multiobjective functions as the power loss reduction and voltage stability improvements with voltage profile and the power balance as constraints. The multi-objective performance index was presented in Ref. [10] to improve the voltage profile and reduces the system losses. In [11], the crisscross optimal algorithm and Monte Carlo simulation was proposed to minimize the total cost and power losses. In [12], the authors suggested an improved particle swarm optimization (IPSO) algorithm for minimizing network loss, electricity price, and operation cost. The improved analytical method was recommended by authors in Ref. [13] to calculate the optimal size of four different DG typologies by using a methodology able to minimize (please insert objective functions). In [14], the optimum sizes and locations of DG units have been found by considering the power losses and voltage profile as objective functions.

In addition, shunt capacitors (SCs) can be used to reduce the variability of some DGs based on renewable energy sources, limit the voltage rise effect, and improve the power quality. Therefore, determining the optimal location and sizing of (SC) when having the integration of DGs in distribution networks has also been considered in literatures. Optimal SCs placement and sizing to improve the bus voltage magnitude and reduce power losses was described by using Genetic Algorithms (GA) implemented in the ETAP software [15]. In [16], a Genetic Algorithm combined with a sequential quadratic programming-based algorithm has been proposed for minimizing the sum of costs, power losses, network upgrading, carrying out the reactive power service, and installation costs. In [17], a load concentration factor based analytical method was proposed to find the optimal solution able for minimizing power losses and improving the voltage profile.

In this paper, a relevant method for determining the optimal location and size of DGs and SCs and operating power factor for each DG is proposed to minimize power losses and enhance the voltage profile in the distribution systems. This proposed method is developed on the Genetic Algorithm (GA) to solve optimal problem based on the nonlinear objective function with discrete and continue variables. The main original contributions of this paper rely on the ability of the method to deal with DGs to control the reactive power injected in the network by determining the optimal power factor (PF) of DGs while considering the contribution also of the SCs and other limitation on the maximum installed capacity of the DGs. These issues, in fact, become crucial in modern distribution networks where the DGs are requested to arrange PF with both the reactive power production and consumption.

The remainder of the paper is organized as follows: Section 2, the proposed method is presented in the form of an optimization model. Some considerations on the GA are detailed in Section 3. In Section 4, with reference to different case studies, the results of numerical experiments are detailed and compared. The conclusions are drawn in the last section.

\section{The Proposed Method}

\subsection{The Objective Function}

The determination of the optimal placement and sizing of DGs and SCs and optimal operating power factor of each DG is obtained by solving a nonlinear minimization 
problem, which is subjected to both equality and inequality constraints and included either discrete and continues variables. The optimization problem can be considered as follows:

$\min f(X)=\min \sum_{i=1}^{N_{b r}} R_{i} I_{i}^{2}$,

where $N_{b r}$ is the number of branches, ${ }^{R_{i}}$ and $I_{\mathrm{i}}$ are the line resistance line current of the branch $i^{\text {th }}$, respectively, and $\mathrm{X}$ is the vector of the optimization variables.

The vector of the optimization variables is:

$X=\left[L O C_{D G}, P_{D G}, P F_{D G}, L O C_{C}, Q_{C}\right]$,

where $L O C_{D G}$ and $L O C_{C}$ are the vectors of the locations (busses) of DGs and SCs, respectively, that can belong to the candidate set of busses defined as:

$L O C_{D G}=\left[L O C_{D G}^{1}, \quad L O C_{D G}^{2}, \ldots, L O C_{D G}^{n}\right]$,

$L O C_{C}=\left[L O C_{C}^{1}, \quad L O C_{C}^{2}, \ldots, \quad L O C_{C}^{m}\right]$.

$P F_{D G}$ is the power factor vector of $\mathrm{DG}_{\mathrm{S}}$ and it is a continuous variable, which has to be included within a minimum $\left({ }^{P F_{D G}^{\min }}\right)$ and maximum value $\left({ }_{D G}^{\max }\right)$ that depend on the DG typology:

$P F_{D G}^{\min } \leq P F_{D G} \leq P F_{D G}^{\max }$,

$P_{D G}$ and $Q_{C}$ are the sizing vectors of $\mathrm{DG}_{\mathrm{S}}$ and SCs, respectively, that can belong to the candidate set of sizes defined as

$$
\begin{aligned}
& P_{D G}=\left[\begin{array}{llll}
P_{D G}^{1}, & P_{D G}^{2}, & \ldots, & P_{D G}^{n_{1}}
\end{array}\right], \\
& Q_{C}=\left[\begin{array}{llll}
Q_{C}^{1}, & Q_{C}^{2}, & \ldots, & Q_{C}^{m_{1}}
\end{array}\right] .
\end{aligned}
$$

\subsection{Constraints}

\subsubsection{Power Flow Equations}

In the optimization system (1), the constraints of the balance power flow are always satisfied, and cam be defined as follows:

$$
\begin{aligned}
& P_{i}=V_{i} \sum_{j=1}^{N} V_{j}\left[G_{i j} \cos \left(\delta_{i}-\delta_{j}\right)+B_{i j} \sin \left(\delta_{i}-\delta_{j}\right)\right], \\
& Q_{i}=V_{i} \sum_{j=1}^{N} V_{j}\left[G_{i j} \sin \left(\delta_{i}-\delta_{j}\right)-B_{i j} \cos \left(\delta_{i}-\delta_{j}\right)\right],
\end{aligned}
$$

where $N$ is the number of nodes in the network, ${ }^{P_{i}}$ and $Q_{i}$ are the active and reactive power of the load $i^{\text {th }}$, respectively, $V_{i}$ is the voltage at node $i^{\text {th }}, G_{i j}$ and $B_{i j}$ are the conductance and susceptance between node $i^{\text {th }}$ and node $j^{\text {th }}$, respectively, $\delta_{i}$ and $\delta_{j}$ are the voltage phase of node $i^{\text {th }}$ and node $j^{\text {th }}$, respectively.

From Eq. (8), the magnitudes and phase-angles of the voltages at buses is determined by using power flow method. Then, the line currents, power flows, power losses, etc. are established to evaluate the constraints.

\subsubsection{Node Voltages}

We consider a constraint of the voltage at node $i^{\text {th }}$ as follows:

$V_{\min } \leq V_{i} \leq V_{\max }, \quad i=1, \ldots, N$,

where $V_{i}$ is the voltage profile at node $i^{\text {th }}$. 


\subsubsection{Thermal Limits}

In order to limit the current flow in the transmission line, we consider the constraints of current as follows:

$I_{i} \leq I_{i}^{\max }, \quad i=1, \ldots, N_{b r}$,

where $I_{i}$ and $I_{i}^{\max }$ are the current magnitude and ampacity of the branch $i^{\text {th }}$, respectively.

\subsubsection{Size of DGs and SCs}

We consider constraints on the injected reactive and active power of DG and SC as follows:

$$
\begin{aligned}
& Q_{D G_{g}}^{\min } \leq Q_{D G_{g}} \leq Q_{D G_{g}}^{\max }, \\
& \sum_{g=1}^{N_{D G}} P_{D G_{g}} \leq k \sum_{i=1}^{N} P_{D_{i}}, \\
& \sum_{g=1}^{N_{D G}} Q_{D G_{g}}+\sum_{s=1}^{N_{C}} Q_{C s} \leq \sum_{i=1}^{N} Q_{D_{i}}, i=1, \ldots, N, g=1, \ldots, N_{D G}, s=1, \ldots, N_{C} \\
& \quad P_{D G_{g}} \text { and } Q_{D G_{g}}=P_{D G_{g}} \tan \left(\arccos \left(P F_{D G_{g}}\right)\right) \text { are the active and reactive power of }
\end{aligned}
$$
the DG $g^{\text {th }}$, respectively; $Q_{D G_{g}}^{\min }$ and $Q_{D G_{g}}^{\max }$ are the minimum and maximum reactive power of the DG $g^{\text {th }}$, respectively; $P_{D_{i}}$ and $Q_{D_{i}}$ are the active and reactive power of load at node $i^{\text {th }}$, respectively; $Q_{C_{s}}$ is the reactive power of the $\mathrm{SC} s^{\text {th }}$, respectively; $k$ is a limiting constant of the injected power having range from zero to $1.0 ;{ }^{N_{D G}}$ and ${ }^{N_{C}}$ are the number of DG and SC, respectively.

\subsubsection{Location of DGs and SCs}

Eventually, a constraint can be imposed also on the location of DGs and SCs that cannot be connected to the same bus:

$$
L O C_{D C} \neq L O C_{C} \text {. }
$$

\section{Optimizing Placement and Size for DGs and SCs, and PF for DGs}

In order to determine the optimal placement and size of DGs and SCs and the optimal operating power factor for each DG, the above-mentioned minimization problem is analyzed and calculated by using Genetic Algorithm (GA). The flowchart of the proposed algorithm is shown in Figure 1. 


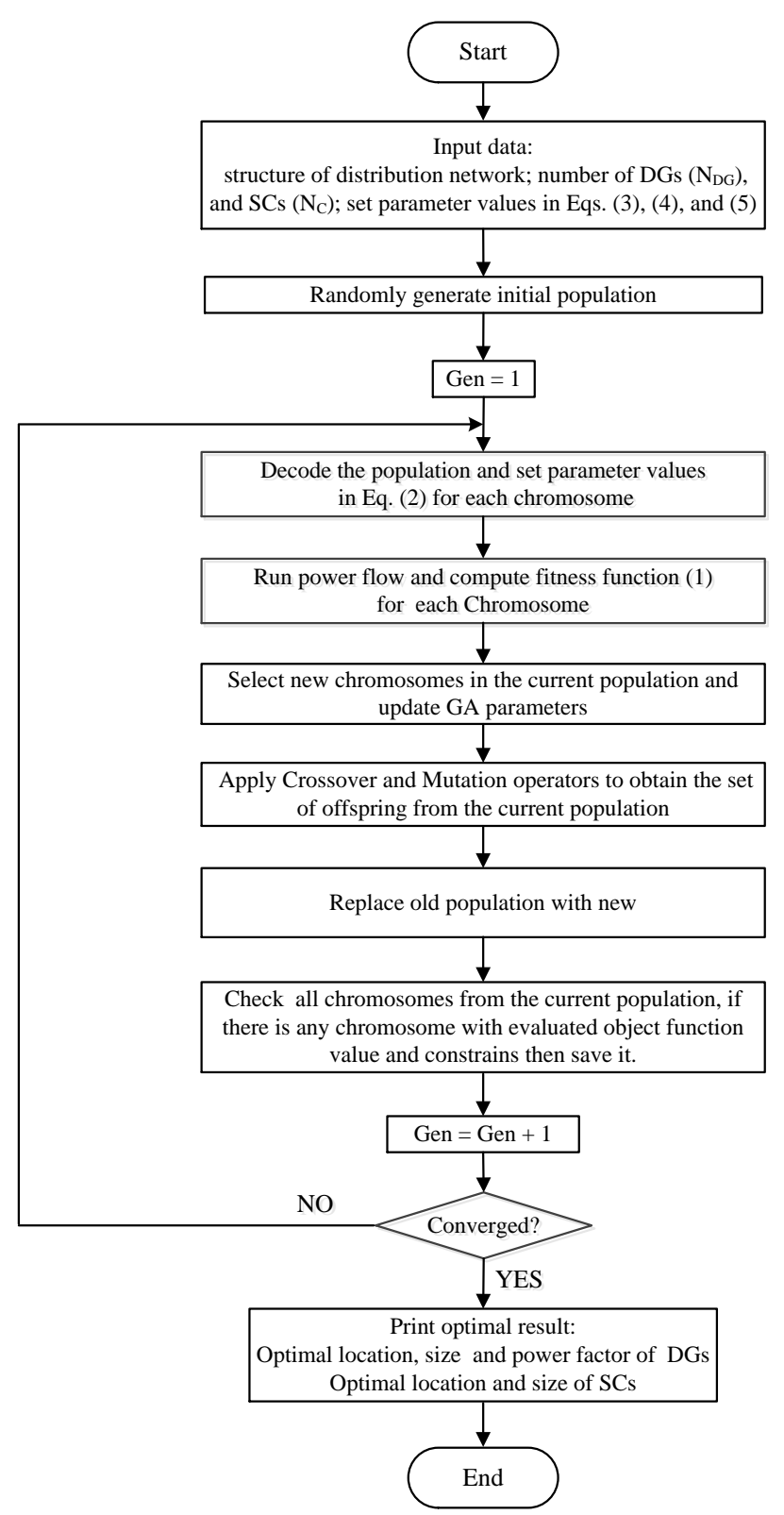

\section{Figure 1. Flowchart Using GA for the Optimization Process}

Details on the flowchart of Figure 1 are given in what fallows:

First. Start.

Step 1. Input data including the structure of distribution network, number of DGs $\left(\mathrm{N}_{\mathrm{DG}}\right)$ and $\mathrm{SCs}\left(\mathrm{N}_{\mathrm{C}}\right)$, and set parameter values in Eqs. (3)-(5).

Step 2. Randomly generate initial population.

Step 3. Set initial counter with Gen $=1$

Step 4. Decode the initial population and set parameter values in Eq. (2) for each chromosome.

Step 5. Run power flow and compute fitness function (1) for each Chromosome.

Step 6. Select the Chromosomes in the current population and update GA parameters.

Step 7. Crossover and mutation: Evaluate the fitness of all of the individuals in the population, and create a new population by performing operation such as the crossover, reproduction, and mutation on the individuals whose fitness has just been measured.

Step 8. Discard the old population and iterate using the new population. 
Step 9. Check all chromosomes from the current population, if there is any chromosome with Evaluate object function value and constrains then save it.

Step 10. Set Gen $=$ Gen +1 .

Step 11. Converged: If all chromosomes are the same or the maximum number of iterations is achieved then save optimal result, else go to Step 4.

Finally. End.

\section{Numerical Experiments}

The IEEE 37-node radial distribution system [18] is considered to take the simulation test, as shown in Figure 2. All dynamic models such as generators, excitation systems, transmission lines, and loads are modeled by using Matlab software. The rated voltage, total active power, and reactive power of the network are $4.8 \mathrm{kV}, 4980 \mathrm{~kW}$, and 1350 $\mathrm{kVAR}$, respectively, corresponding the total active power loss is $220.60 \mathrm{~kW}$ and the total reactive power loss is $200.18 \mathrm{kVAR}$ in the base case without DGs and SCs. In that case, the minimum voltage magnitude is 0.9221 p.u. at node 36 and the maximum voltage magnitude is 1.00 p.u. at node 1 .

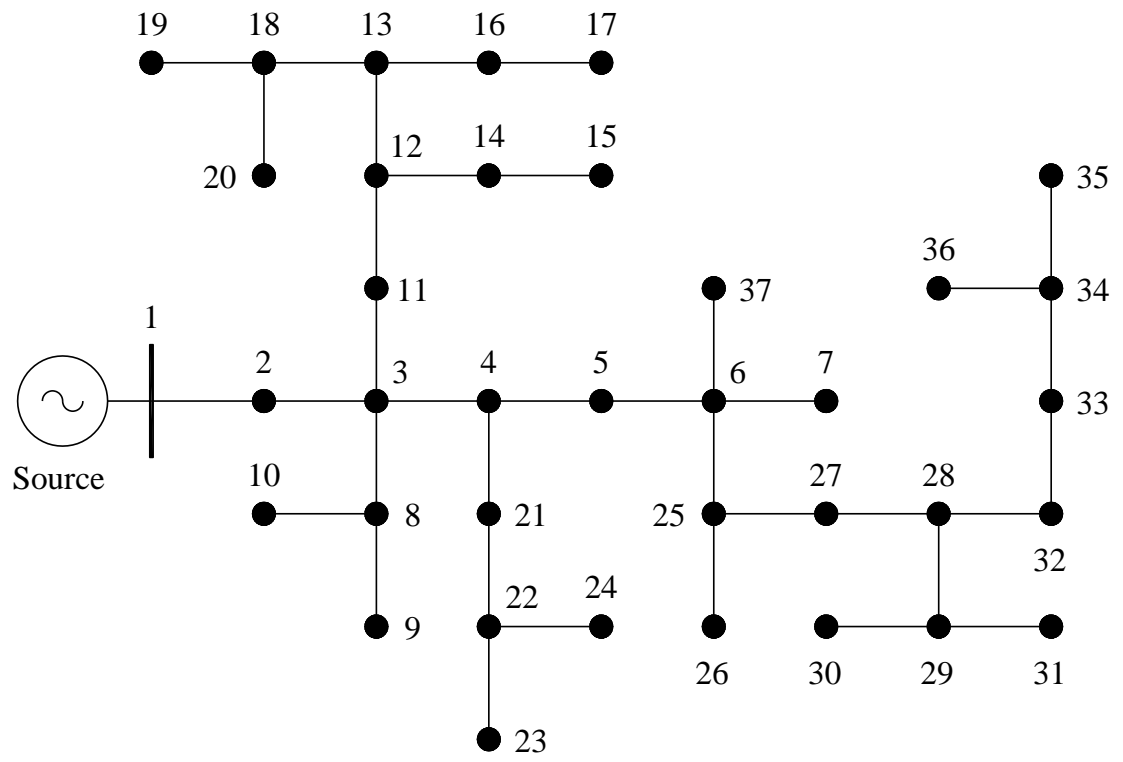

Figure 2. The IEEE 37-node Network [18]

Using the proposed algorithm in Section 3, the desired number of DGs to place is four units and the desired number of SCs to place four too. We consider that the candidate sizes of each DG ranges from $200 \mathrm{~kW}$ to $5000 \mathrm{~kW}$ and each changed step is supposed to be $50 \mathrm{~kW}$. DGs can generate or absorb the reactive power at their different power factors having range between 0.7 and 1.0. Similarly, the candidate sizes each SC ranges from 25 $\mathrm{kVAR}$ to $300 \mathrm{kVAR}$ in which each changed step is supposed to be $25 \mathrm{kVAR}$.

\subsection{Optimization with Different Values of $k$ Factor}

In this subsection, the proposed method was applied with reference to different values of $k$ factor in order to consider that the total power supplied by DGs is not more than a specified percentage of total demand. It is consequent for the real networks when we want to limit the active power of DGs for connecting. The optimization results with case $k=$ 1.0 , case $k=0.7$, and case $k=0.5$ are been showed in Table 1 . 
Table 1. The Optimization Results with Different Values of $K$ Factor

\begin{tabular}{|c|c|c|c|c|c|}
\hline \multirow{13}{*}{$k=1.0$} & & \multicolumn{4}{|c|}{ DG's location and size } \\
\hline & DG's location & 21 & 12 & 31 & 28 \\
\hline & DG's size $(\mathrm{kW})$ & 800 & 1450 & 350 & 1300 \\
\hline & Power factor (PF*) & 0.9846 & 0.9873 & 0.9505 & 0.9912 \\
\hline & \multicolumn{5}{|c|}{ SC's location and size } \\
\hline & SC's location & 13 & 8 & 32 & 22 \\
\hline & SC's size (kVAR) & 275 & 75 & 125 & 75 \\
\hline & & \multicolumn{4}{|c|}{ Loss } \\
\hline & Active power loss $(\mathrm{kW})$ & \multicolumn{4}{|c|}{19.03} \\
\hline & Reactive power loss (kVAR) & \multicolumn{4}{|c|}{11.79} \\
\hline & & \multicolumn{4}{|c|}{ Voltage profile } \\
\hline & Minimum voltage (p.u) & \multicolumn{4}{|c|}{0.99} \\
\hline & Maximum voltage (p.u) & \multicolumn{4}{|c|}{1.00} \\
\hline \multirow{13}{*}{$k=0.7$} & & \multicolumn{4}{|c|}{ DG's location and size } \\
\hline & DG's location & 32 & 21 & 19 & 6 \\
\hline & DG's size $(\mathrm{kW})$ & 1300 & 500 & 850 & 650 \\
\hline & Power factor & 0.9912 & 0.9917 & 0.9803 & 0.9515 \\
\hline & & \multicolumn{4}{|c|}{ SC's location and size } \\
\hline & \multirow{3}{*}{$\begin{array}{l}\text { SC's location } \\
\text { SC's size (kVAR) }\end{array}$} & \multirow{2}{*}{\multicolumn{2}{|c|}{$\begin{array}{c}18 \\
125 \\
\end{array}$}} & 31 & 33 \\
\hline & & & & 175 & 75 \\
\hline & & \multicolumn{4}{|c|}{ Loss } \\
\hline & Active power loss $(\mathrm{kW})$ & \multicolumn{4}{|c|}{20.8441} \\
\hline & Reactive power loss (kVAR) & \multicolumn{4}{|c|}{15.3539} \\
\hline & & \multicolumn{4}{|c|}{ Voltage profile } \\
\hline & Minimum voltage (p.u) & \multicolumn{4}{|c|}{0.9893} \\
\hline & Maximum voltage (p.u) & \multicolumn{4}{|c|}{1.0004} \\
\hline \multirow{13}{*}{$k=0.5$} & & \multicolumn{4}{|c|}{ DG's location and size } \\
\hline & DG's location & 33 & 27 & 20 & 14 \\
\hline & DG's size $(\mathrm{kW})$ & 950 & 900 & 250 & 200 \\
\hline & Power factor & 0.988 & 0.9087 & 0.8863 & 0.8033 \\
\hline & & \multicolumn{4}{|c|}{ SC's location and size } \\
\hline & SC's location & 34 & 21 & 24 & 5 \\
\hline & SC's size (kVAR) & 50 & 75 & 50 & 200 \\
\hline & & \multicolumn{4}{|c|}{ Loss } \\
\hline & Active power loss $(\mathrm{kW})$ & \multicolumn{4}{|c|}{42.2733} \\
\hline & Reactive power loss (kVAR) & & & & \\
\hline & & & Volta & rofile & \\
\hline & Minimum voltage (p.u) & & & & \\
\hline & Maximum voltage (p.u) & & & & \\
\hline
\end{tabular}

From Table 1, the optimal results have been summarized as:

Case 1: $k=1$

The total active power of DGs is $3900 \mathrm{~kW}$ installed at nodes 21, 12, 31, and 28; whereas, the SCs are installed at nodes $13,8,32$ and 22. The active and reactive power losses are $19.0338 \mathrm{~kW}$ and $11.7880 \mathrm{kVAR}$, respectively.

Case 2: $k=0.7$

The total active power of DGs is $3300 \mathrm{~kW}$ installed at nodes 32, 21, 19, and 16; whereas, the SCs are also installed at node 18,20,31 and 33 in the network. The active and reactive power losses are $20.8441 \mathrm{~kW}$ and $15.3539 \mathrm{kVAR}$, respectively.

Case 3: $k=0.5$

The total active power of DGs is $2300 \mathrm{~kW}$ installed at nodes 33, 21, 19 and 6; whereas, the SCs are installed at nodes 34, 21, 24 and 5. The active and reactive power losses are $42.2733 \mathrm{~kW}$ and $37.7385 \mathrm{kVAR}$, respectively. 
From these results, the proposed method proves the optimal placement and size of DGs and SCs and the optimal operating power factor for each DG. The real values of $k$ factors in case 1 , case 2 , and case 3 are $k_{1}=0.783, k_{2}=0.662$, and $k_{3}=0.462$, respectively. The $k_{2}$ and $k_{3}$ factors are lower than their set values because they depend on the initial set of DG's size. The $k_{l}$ factor is optimal factor $\left(k=k^{*}\right)$ because the active power loss in this case is least.

Observationally, from Table 1, it can be shown that the locations of DGs are different with the locations of SCs, which is suitable for the installation of DGs and SCs in the distribution network.

Figure 3 shows the results of voltage profiles after optimization for different values of $k$ factor. It can be observed that the voltage profiles are significantly improved in Case 1 .

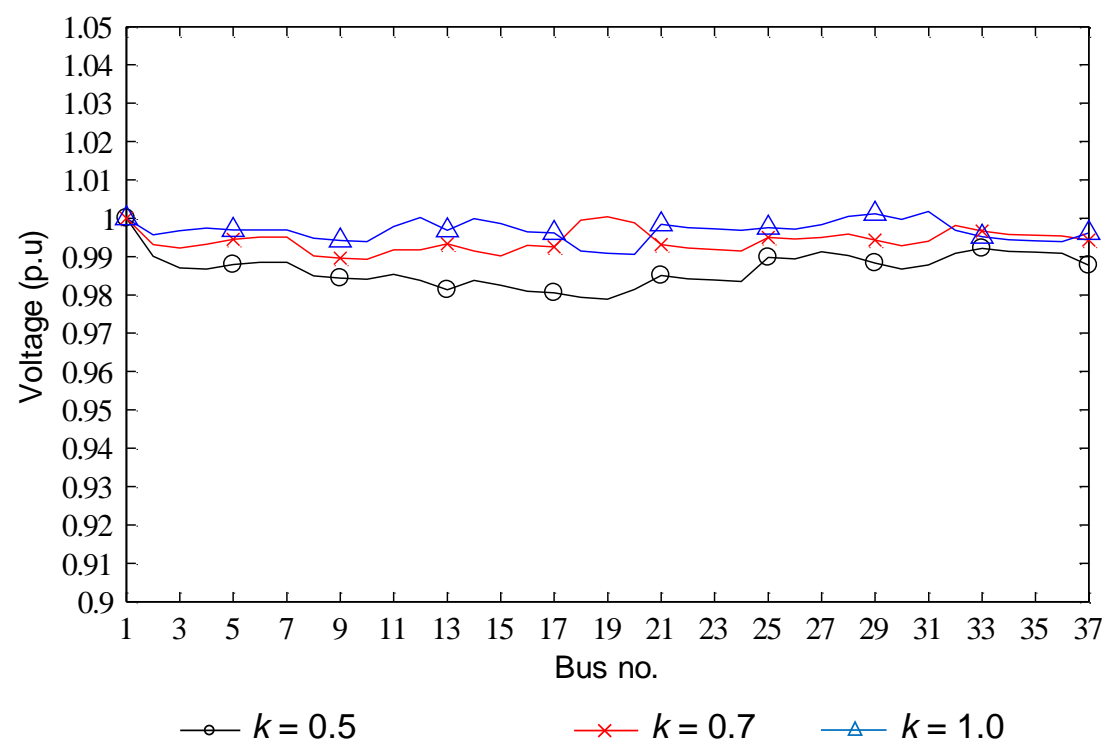

Figure 3. Voltage Profiles with Different Values of $K$ Factor

Basing on the results in Table 1 and Figure 3, we can conclude that the optimal placements of DGs are nodes 12, 21, 28, and 31 corresponding to sizes 1450, 800, 1300, and $350 \mathrm{~kW}$; and optimal power factors $\left(\mathrm{PF}^{*}\right)$ of $0.9873,0.9846,0.9912$, and 0.9505 , respectively. Similarly, the optimal placements of SCs are nodes 8, 13, 22, and 32 corresponding to sizes $75,275,75$, and $125 \mathrm{kVAR}$, respectively. In addition, if the total power supplied by DG is limited to equal the total demand then the proposed method will obtain the best results for minimization power losses and voltage profile improvement.

\subsection{Verifying Optimal Power Factor}

In this subsection, our main objective is to re-check the optimal power factor ( $\left.\mathrm{PF}^{*}\right)$ of DGs. In order to do this, we suppose that the optimal placement and size of DGs and SCs, as concluded above, are carried to test based on the different PFs of 0.7, 0.8, 0.9, and 1.0. Note that four DGs are operating at the same power factor. Figure 4 plosts the total power losses of network. 


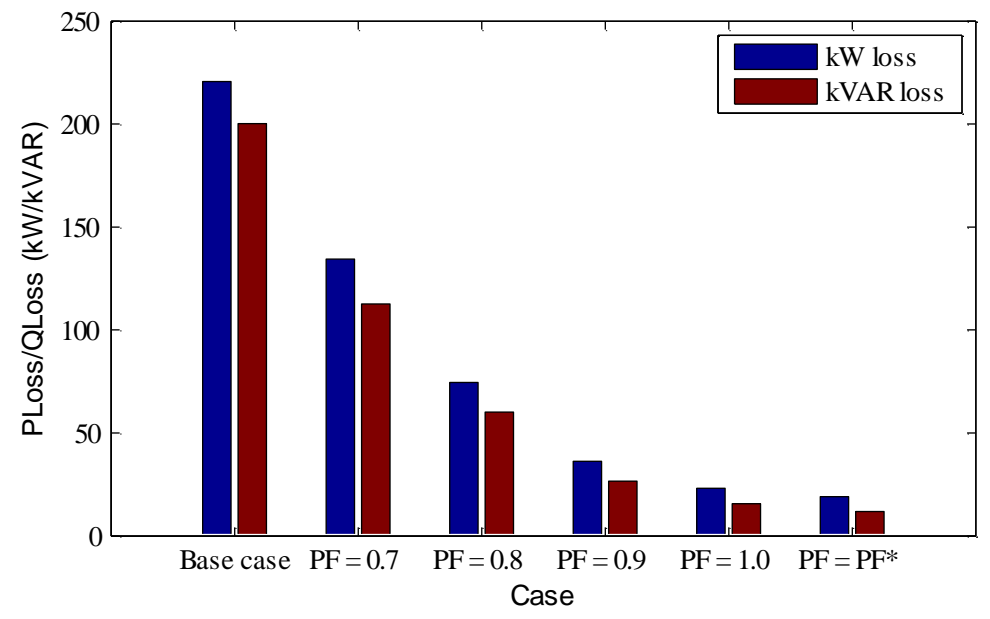

Figure 4. The Total Power Losses with the different PF $_{s}$

As can be observed from Figure 4, the total power loss is the smallest corresponding to the optimal power factor ( $\left.\mathrm{PF}^{*}\right)$. Therefore, the conclusion in Section 4.1 for the optimal power factors is confirmed.

\subsection{Verifying Optimal Location, Size, and Power Factor}

In this subsection, We want to verify optimal location and size of DGs and SCs and optimal power factor of DGs by this proposed method. We consider the operating modes that are established based on the level of integration of DGs and SCs as follows:

Case (i) with four units of SC and without units of DG,

Case (ii) with four units of DG and without units of SC

Case (iii) with four units of DG and four units of SC.

Case (ii) and Case (iii) are carried out to simulate basing on five different values of power factors for all DGs. The obtained results are listed in Table 2

Table 2. Results for Case Studies (i), (ii) and (iii) for different Values of PFs

\begin{tabular}{|c|c|c|c|c|c|c|c|c|c|c|c|c|}
\hline \multirow{2}{*}{ Case } & & \multirow{2}{*}{$\begin{array}{c}\mathbf{P}_{\mathbf{D}} \\
(\mathbf{k W})\end{array}$} & \multirow{2}{*}{$\begin{array}{c}\mathbf{Q D}_{\mathbf{D}} \\
(\mathrm{kVAR})\end{array}$} & \multirow{2}{*}{$\begin{array}{l}\mathbf{P}_{\mathrm{DG}} \\
(\mathrm{kW})\end{array}$} & \multirow{2}{*}{$\begin{array}{c}\mathbf{Q}_{\mathrm{DG}} \\
(\mathrm{kVAR})\end{array}$} & \multirow{2}{*}{$\begin{array}{c}\mathbf{Q}_{\mathrm{sc}} \\
(\mathrm{kVAR})\end{array}$} & \multirow{2}{*}{$\begin{array}{c}\mathbf{P}_{\text {loss }} \\
(\mathbf{k W})\end{array}$} & \multirow{2}{*}{$\begin{array}{c}\mathbf{Q}_{\text {loss }} \\
(\mathbf{k V A R})\end{array}$} & \multicolumn{2}{|c|}{$\begin{array}{l}\text { voltage profile } \\
\text { (min) }\end{array}$} & \multicolumn{2}{|c|}{$\begin{array}{l}\text { voltage profile } \\
\text { (max) }\end{array}$} \\
\hline & & & & & & & & & $\begin{array}{l}V_{\text {min }} \\
\text { (p.u.) }\end{array}$ & Node & $\begin{array}{l}V_{\max } \\
\text { (p.u.) }\end{array}$ & Node \\
\hline Base & - & 4980 & 1350 & - & - & - & 220.60 & 200.18 & 0.922 & 36 & 1.000 & 1 \\
\hline (i) & - & 4980 & 1350 & - & - & 550 & 208.76 & 189.29 & 0.928 & 36 & 1.000 & 1 \\
\hline \multirow{5}{*}{ (ii) } & & 4980 & 1350 & & & - & 21.25 & 13.92 & 0.985 & 20 & 1.000 & 1 \\
\hline & $\mathrm{PF}=0.7$ & 4980 & 1350 & 3900 & 3978.8 & - & 105.34 & 86.29 & 1.000 & 1 & 1.034 & 31 \\
\hline & $\mathrm{PF}=0.8$ & 4980 & 1350 & 3900 & 2925.0 & - & 54.84 & 42.28 & 1.000 & 1 & 1.023 & 31 \\
\hline & $\mathrm{PF}=0.9$ & 4980 & 1350 & 3900 & 1888.9 & - & 26.30 & 17.67 & 0.994 & 20 & 1.011 & 31 \\
\hline & $\mathrm{PF}=1.0$ & 4980 & 1350 & 3900 & - & - & 32.29 & 24.07 & 0.980 & 20 & 1.000 & 1 \\
\hline \multirow{5}{*}{ (iii) } & $\mathrm{PF}=\mathrm{PF}^{*}$ & 4980 & 1350 & 3900 & 663.9 & 550 & 19.03 & 11.79 & 0.991 & 20 & 1.002 & 31 \\
\hline & $\mathrm{PF}=0.7$ & 4980 & 1350 & 3900 & 3978.8 & 550 & 134.49 & 112.66 & 1.000 & 1 & 1.039 & 31 \\
\hline & $\mathrm{PF}=0.8$ & 4980 & 1350 & 3900 & 2925.0 & 550 & 74.59 & 60.10 & 1.000 & 1 & 1.027 & 31 \\
\hline & $\mathrm{PF}=0.9$ & 4980 & 1350 & 3900 & 1888.9 & 550 & 36.31 & 26.64 & 1.000 & 20 & 1.016 & 31 \\
\hline & $\mathrm{PF}=1.0$ & 4980 & 1350 & 3900 & - & 550 & 23.07 & 15.58 & 0.986 & 20 & 1.000 & 1 \\
\hline
\end{tabular}

Note: $\mathrm{PF}=\mathrm{pf}$ and $\mathrm{PF}=\mathrm{PF}^{*}$ : This is $\mathrm{PFs}$ of DGs is determined for optimization having range between 0.7 and 1.0 
Table 2 shows that the case (iii) has the smallest total active and reactive power losses, so that the optimal placement and size of DGs and SCs and optimal power factor of each DG is satified as concluded in Setion 4.1.

In addition, Figure 5 plots the voltage profile for case (iii). It is evident that the voltage profile at optimal power factor is the best.

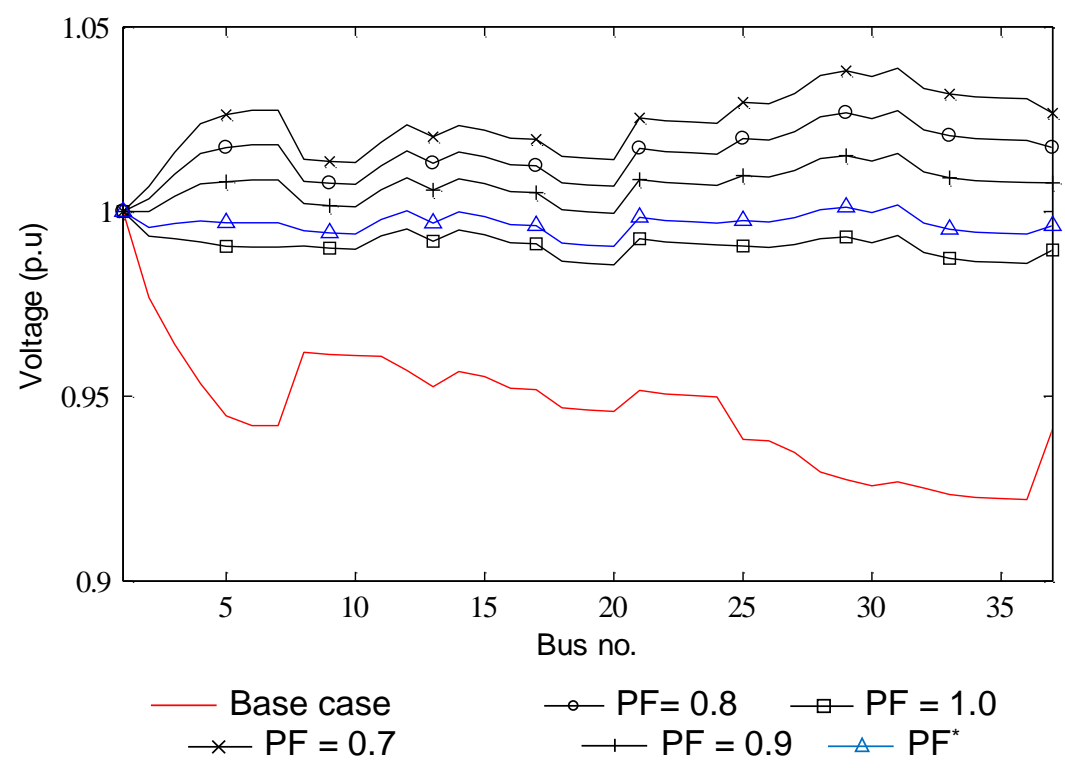

Figure 5. Voltage Profiles for Case (lii) with Different Values of Pfs

The active power losses on the lines are depending on the current magnitude. From the power flow results at different operation modes as mentioned, the current magnitudes on some lines significantly change as shown in Figure 6.

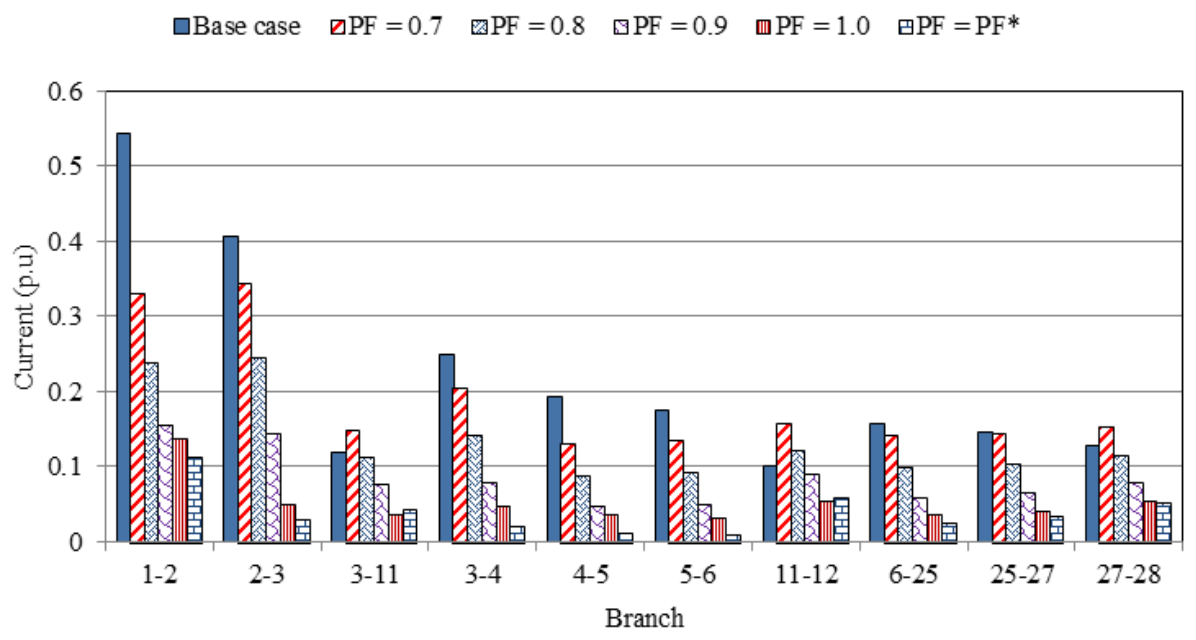

Figure 6. The Current Values for Case (lii) with Different Values of Pfs

In Figure 6, for case of $\mathrm{PF}=0.7$ the current magnitudes in the lines are higher than in other cases except the base case, so the same happens for the total power losses. On the contrary, in the case DGs operated at the optimal power factors $\left(\mathrm{PF}=\mathrm{PF}^{*}\right)$ the current magnitudes on the lines are lower than in the other cases. 


\section{Conclusion}

The paper has proposed a relevant method to plane the optimal placement and size of DGs and SCs and operating power factor for each DG in distribution systems. The method is developed basing on the Genetic Algorithm (GA) to minimize power losses and enhance the voltage profile in distribution systems.

The IEEE 37-node radial distribution system has been considered for numerous numerical experiments applying the proposed method. The optimal placement and size of DGs and SCs and operating power factor for each DG have been determined. With reference to different scenarios, the results have been detailed and compared proving that the optimization for placement and size of DGs and SCs and operating power factor for each DG always get the best results. Therefore, the proposed method seems characterized by high effectiveness and robustness.

\section{Acknowledgments}

The authors sincerely acknowledge the financial support provided by the Erasmus mundus programmer of the European Commission. This research has finished at the University of Campania (former Second University of Napoli), L.Vanvitelli, Italy from project of EMMAsia 2014 (Erasmus Mundus Mobility with Asia) under the supervision of Prof. Alfredo Testa.

\section{References}

[1] Shipeng Du, Qianzhi Shao and Gang Wang, "Analysis of DG Influences on System Losses in Distribution Network", International Journal of Grid Distribution Computing, vol. 8, no.5, (2015), pp. 141-152.

[2] Hong Liu, Yinchang Guo, Shaoyun Ge, and Mingxin Zhao, "Impact of DG Configuration on Maximum Use of Load Supply Capability in Distribution Power Systems", Hindawi Publishing Coporation Journal of Applied Mathematics, vol. 2014, (2014), article ID 136726.

[3] Soo-Hyoung Lee and Jung-Wook Park, "Selection of Optimal Location and Size of Multiple Distributed Generations by Using Kalman Filter Algorithm”, IEEE Transactions on Power Systems, vol. 24, no. 3, (2009), pp. 1393-1400.

[4] Soo Hyoung Lee and Jung-Wook Park, "Optimal Placement and Sizing of Multiple DGs in a Practical Distribution System by Considering Power Loss", IEEE Transactions on Industry Applications, vol. 49, no. 5, (2013), pp. 2262-2270.

[5] Vasileios A. Evangelopoulos, Pavlos S. Georgilakis, "Optimal distributed deneration placement under uncertainties based on point estimate method embedded genetic algorithm", IET Gener. Transm. Distrib, vol. 8, Iss. 3, (2014), pp. 389-400.

[6] Juan Andrés Martín García, Antonio José Gil Mena, "Optimal distributed generation location and size using a modified teaching-learning based optimization algorithm", Electrical Power and Energy Systems, vol 50, (2013), pp. 65-75.

[7] Aggelos S. Bouhouras, Kallisthenis I. Sgouras, Paschalis A. Gkaidatzis, Dimitris P. Labridis, "Optimal active and reactive nodal power requirements towards loss minimization under reverse power flow constraint defining DG type", Electrical Power and Energy Systems, vol 78, (2016), pp. 445-454.

[8] Mohammad H. Moradi, S.M. Reza Tousi, Mohammad Abedini, "Multi-objective PFDE algorithm for solving the optimal siting and sizing problem of multiple DG sources", Electrical Power and Energy Systems, vol 56, (2014), pp. 117-126.

[9] Kumar Mahesh, Perumal Nallagownden and Irraivan Elamvazuthi, "Advanced Pareto Front NonDominated Sorting Multi-Objective Particle Swarm Optimization for Optimal Placement and Sizing of Distributed Generation”, Energies, 9, 982, (2016), pp. 2-23.

[10] N. Mohandas, R. Balamurugan, L. Lakshminarasimman, "Optimal location and sizing of real power DG units to improve the voltage stability in the distribution system using ABC algorithm united with chaos", Electrical Power and Energy Systems, vol. 66, (2015), pp. 41-52.

[11] Xiangang Peng, Lixiang Lin, Weiqin Zheng and Yi Liu, "Crisscross Optimization Algorithm and Monte Carlo Simulation for Solving Optimal Distributed Generation Allocation Problem”, Energies, 8, (2015), pp. 13641-13659.

[12] Zeng Pin-zhuo, Wang Ke-you, Li Guo-jie and Jiang Xiu-chen, "Optimization of Distributed Generation Integrated into Micro Grids Considering the Correlation of DGs", International Journal of Grid Distribution Computing, vol 8, no.6, (2015), pp. 105-116. 
[13] Duong Quoc Hung, and Nadarajah Mithulananthan, "Multiple Distributed Generator Placement in Primary Distribution Networks for Loss Reduction", IEEE Transactions on Industrial Electronics, vol. 60, no. 4, (2013), pp. 1700-1708.

[14] Komail Nekooei, Malihe M. Farsangi, Hossein Nezamabadi-Pour, and Kwang Y. Lee, "An Improved Multi-Objective Harmony Search for Optimal Placement of DGs in Distribution Systems", IEEE Transactions on Smart Grid, vol. 4, no. 1, (2013), pp. 557-567.

[15] Qasim Kamil Mohsin , Xiangning Lin, Firas F.M. Flaih, Samir M. Dawoud, Mohammed Kdair, "Optimal Placement and Capacity of Capacitor Bank in Radial Distribution System", International Conference on Energy Efficient Technologies for Sustainability (ICEETS), (2016), pp. 416-423.

[16] G. Carpinelli, F. Mottola, D. Proto, A. Russo, "Optimal Allocation of Dispersed Generators, Capacitors and Distributed Energy Storage Systems in Distribution Networks", Modern Electric Power Systems (2010), pp. 1-6.

[17] Mohsin Shahzad, Ishtiaq Ahmad, Wolfgang Gawlik, and Peter Palensky, "Load Concentration Factor Based Analytical Method for Optimal Placement of Multiple Distribution Generators for Loss Minimization and Voltage Profile Improvement”, Energies, 9, 287, (2016), pp. 1-21.

[18] Website: http://ewh.ieee.org/soc/pes/dsacom/testfeeders/.

\section{Authors}

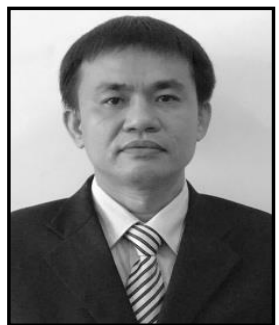

Doan Duc Tung, received MS and $\mathrm{PhD}$ degrees in Electrical Engineering from the Ha Noi University of Science and Technology, Ha Noi, Viet Nam, in 2004 and 2009, respectively. He is working at Quy Nhon University as lecturer and he is currently postdoctoral research at the Department of Industrial and Information Engineering, University of Campania, L. Vanvitelli, Italy. His research interests are Electrical Machines, Optimization Techniques in Electrical Machines and Power Systems, Smart Grids.

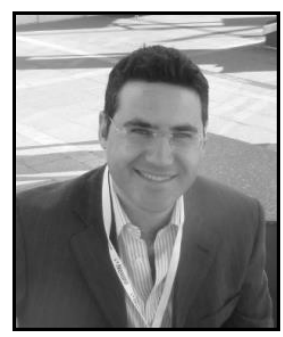

Fabio Mottola, received the Laurea Cum Laude and $\mathrm{PhD}$ degrees both in Electrical Engineering from the University of Naples, Federico II, Napoli, Italy, in 2004 and 2008, respectively. His main research interests focus on optimization methods for planning and operation of power systems.

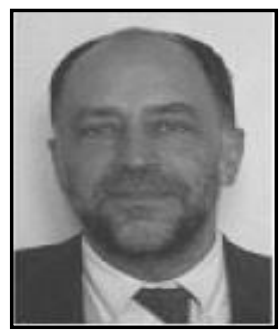

Alfredo Testa, (M'83-SM'03-F'08) was born in Naples, Italy, on March 10, 1950. He received the degree in electrical engineering from the University of Naples, Naples, Italy, in 1975. Currently, he is a Professor in Electrical Power Systems at the University of Campania, L.Vanvitelli, Italy. He is engaged in research on electrical power systems reliability and harmonic analysis. Dr. Testa is a Fellow member of the IEEE Power Engineering Society and the Italian Institute of Electrical Engineers (AEI). 